\title{
Weak admissibility does not imply admissibility for analytic semigroups
}

\author{
Hans Zwart ${ }^{a}$ Birgit Jacob ${ }^{b}$ Olof Staffans ${ }^{c}$ \\ a University of Twente \\ Faculty of Mathematical Sciences \\ P.O. Box 217 \\ NL-7500 AE Enschede \\ The Netherlands \\ h.j.zwart@math.utwente.nl \\ b School of Mathematics \\ University of Leeds \\ Leeds LS2 9JT \\ $U K$ \\ birgit@amsta.leeds.ac.uk \\ c Abo Akademi University \\ Department of Mathematics, \\ FIN-20500 Abo \\ Finland \\ Olof.Staffans@abo.fi
}

\begin{abstract}
Two conjectures on admissible control operators by George Weiss are disproved in this paper. One conjecture says that an operator $B$ defined on an infinite-dimensional Hilbert space $U$ is an admissible control operator if for every element $u \in U$ the vector $B u$ defines an admissible control operator. The other conjecture says that $B$ is an admissible control operator if a certain resolvent estimate is satisfied. The examples given in this paper show that even for analytic semigroups the conjectures do not hold. In the last section we construct a semigroup example showing that the first estimate in the Hille-Yosida theorem is not sufficient to conclude boundedness of the semigroup.
\end{abstract}

Key words: Infinite-dimensional system, admissible control operator, conditional basis, $C_{0}$-semigroup. 


\section{Introduction}

It is well-known that homogeneous linear partial differential equations can be written as abstract differential equations on a Banach or Hilbert space. For instance, the diffusion equation in a metal bar of length one,

$$
\frac{\partial}{\partial t} w(t, \xi)=\frac{\partial^{2}}{\partial \xi^{2}} w(t, \xi), \quad t \geq 0, \quad \xi \in(0,1)
$$

with boundary conditions

$$
\frac{\partial}{\partial \xi} w(t, 0)=\frac{\partial}{\partial \xi} w(t, 1)=0
$$

can be written as the abstract differential equation

$$
\dot{x}(t)=A x(t)
$$

where $x(t)$ denotes the temperature profile at time $t$, i.e., $w(t, \cdot)$. This temperature profile is assumed to be an element of $L^{2}(0,1)$. Furthermore, $A$ is a linear operator from its domain $D(A)$ to $L^{2}(0,1)$ defined as

$$
A h=\frac{d^{2} h}{d \xi^{2}}
$$

on

$$
D(A)=\left\{h \in L^{2}(0,1) \mid \frac{d^{2} h}{d \xi^{2}} \in L^{2}(0,1) \text { with } \frac{d h}{d \xi}(0)=\frac{d h}{d \xi}(1)=0\right\}
$$

A homogeneous linear partial differential equation (p.d.e.) has for every initial condition a unique (weak) solution which depends continuously on the initial condition if and only if the operator $A$ appearing in the corresponding abstract differential equation generates a $C_{0}$-semigroup, which we denote by $T(\cdot)$. For the partial differential equation (1) with boundary conditions (2) this is the case. Furthermore, $T(t) w(0, \cdot)=x(t)$ in $L^{2}(0,1)$ for all $t \geq 0$. For more detail, see Curtain and Zwart [2, Chapter 2].

The Hille-Yosida theorem gives necessary and sufficient conditions for an operator $A$ to generate a $C_{0}$-semigroup. Hence this theorem can be used to determine whether the p.d.e. has a unique solution which depends continuously on the initial condition. For inhomogeneous partial differential equations George Weiss conjectured similar results ten years ago. Before we formulate 
these conjectures we show how an inhomogeneous p.d.e. can be reformulated as an inhomogeneous differential equation in a Hilbert space.

Consider the p.d.e. (1) with inhomogeneous boundary conditions

$$
\frac{\partial}{\partial \xi} w(t, 0)=u(t), \quad \frac{\partial}{\partial \xi} w(t, 1)=0
$$

Introducing again $x(t)$ as the temperature profile at time $t$, we can rewrite (1) and (4) as (see Salamon [12])

$$
\dot{x}(t)=A x(t)+B u(t), \quad x(0)=x_{0}, \quad t \geq 0,
$$

where $A$ is the same as in (3) and $B$ is given as

$$
B=\delta
$$

where $\delta$ is the delta distribution at $\xi=0$. Hence we see that $B$ does not lie in $L^{2}(0,1)$, and thus does not define an operator from $\mathbb{C}$ to $L^{2}(0,1)$. Since $L^{2}(0,1)$ is the space in which we want that the state $x(t)$ takes its values, it is not directly clear if every input function $u$ is such that the solution $x$ of (5) lies in $L^{2}(0,1)$. As in the Hille-Yosida theorem, we would like to conclude this from properties of the operators $A$ and $B$.

We consider the abstract differential equation

$$
\dot{x}(t)=A x(t)+B u(t), \quad x(0)=x_{0}, \quad t \geq 0
$$

on a Hilbert space $H$ with $x_{0} \in H$ and with $u$ in some space of functions taking values in a Hilbert space $U$. We denote this class of inputs by $\mathcal{U}$. We wonder if for any input $u \in \mathcal{U}$ and any initial condition $x_{0}$ there exists a unique solution $x$ with values in $H$ satisfying (weakly) the equation (7). Choosing $u=0$, we see that $A$ has to generate a $C_{0}$-semigroup $T(\cdot)$ on $H$. The answer to the earlier question depends on the class $\mathcal{U}$ of input functions. If $u$ is very smooth, then it is more likely that (7) has a solution than if $u$ is merely $L^{1}$. Throughout this paper we take $\mathcal{U}$ to be $L_{\text {loc }}^{2}(0, \infty ; U)$. This choice of input functions is motivated by the fact that this is the space which is normally used in control theory.

Weiss [16] showed that if the solution of (7) takes its values in $H$ for any $u \in L_{\text {loc }}^{2}(0, \infty ; U)$, then $B \in \mathcal{L}\left(U, D\left(A^{*}\right)^{\prime}\right)$, where $D\left(A^{*}\right)$ is the domain of the adjoint of $A$, and ' denotes the dual space. $D\left(A^{*}\right)^{\prime}$ can also be seen as the completion of $H$ with respect to the norm

$$
\|x\|_{D\left(A^{*}\right)^{\prime}}=\left\|(\lambda I-A)^{-1} x\right\|_{H},
$$


where $\lambda$ is an arbitrary point in the resolvent set of $A$. Note that this implies that $B$ is a bounded operator from $U$ to $H$ whenever $A$ is a bounded operator on $H$ (usually $A$ is unbounded).

Since the $C_{0}$-semigroup $T(\cdot)$ can be extended to $D\left(A^{*}\right)^{\prime}$, we can consider $(7)$ as an abstract differential equation on this larger Hilbert space. As an operator from $U$ to $D\left(A^{*}\right)^{\prime}$, the control operator $B$ is bounded and thus the solution (7) is given by

$$
x(t)=T(t) x_{0}+\int_{0}^{t} T(t-\rho) B u(\rho) d \rho
$$

Since $x_{0}$ is an element of $H$, it is clear that $x(t)$ lies in $H$ if and only if the integral term lies in $H$ for every $u$. Operators $B$ for which the integral term lies in $H$ for every $u$ are called admissible.

Definition $1.1 B \in \mathcal{L}\left(U, D\left(A^{*}\right)^{\prime}\right)$ is called an admissible control operator for $T(\cdot)$ if, for some $t>0$,

$$
\int_{0}^{t} T(t-\rho) B u(\rho) d \rho \in H
$$

for all $u \in L^{2}(0, t ; U)$. $B \in \mathcal{L}\left(U, D\left(A^{*}\right)^{\prime}\right)$ is a weakly admissible control operator for $T(\cdot)$, if for every $v \in U, B v$ is an admissible control operator for $T(\cdot)$, i.e., (9) holds for all $u$ of the form $u(\rho)=v w(\rho)$, where $v \in U$ and $w \in L^{2}(0, t ; \mathbb{C})$.

Using the semigroup property of $T(\cdot)$, it is not hard to see that (9) is satisfied for every $t>0$ if it is satisfied for some $t>0$.

It follows form the closed graph theorem that if $B$ is an admissible control operator for $T(\cdot)$, then, for each $t>0$, there exists a constant $M_{t}>0$ such that

$$
\left\|\int_{0}^{t} T(t-\rho) B u(\rho) d \rho\right\|_{H} \leq M_{t}\|u\|_{L^{2}(0, t ; U)}, \quad u \in L^{2}(0, t ; U) .
$$

Thus, an inhomogeneous linear partial differential equation of the type (7) has for every initial condition and every locally square integrable input a unique (weak) solution which depends continuously on the initial condition and the input if and only if the operator $A$ generates a $C_{0}$-semigroup $T(\cdot)$ and $B$ is an admissible control operator for $T(\cdot)$. 
For p.d.e.'s special techniques for solving the admissibility problem are available, see for example Lasiecka and Triggiani [9]. If the operator $A$ has a Riesz basis of eigenvectors and $U=\mathbb{C}$, then it has been proved that admissibility of $B$ is equivalent to the fact that a certain measure is a Carleson measure, see Ho and Russell [7] and Weiss [15]. All these results apply only to specific cases.

Weiss [17] conjectured the following simple condition for the admissibility of $B$.

Conjecture 1.2 Let $B \in \mathcal{L}\left(U, D\left(A^{*}\right)^{\prime}\right)$. Then $B$ is an admissible control operator for $T(\cdot)$ if and only if $B$ is a weakly admissible control operator for $T(\cdot)$.

Clearly, admissibility implies weak admissibility. For left-invertible $C_{0}$-semigroups Weiss [17] showed that also the converse is true, i.e., the conjecture holds for such semigroups. This implication has also been proved for normal analytic semigroups, see Hansen and Weiss [6]. Here we show that this implication no longer holds for compact analytic semigroups, see Example 2.3. It is a little bit surprising that the conjecture does not hold for analytic semigroups, since they satisfy $T(t) B \in \mathcal{L}(U, H)$ for all $t>0$.

Let $\omega$ denote the growth bound of $T(\cdot)$. Taking $u(t)=e^{-s t} u_{0}$ with $u_{0} \in U$ and $\operatorname{Re}(s)>\max \{0, \omega+1\}$, and using (10) we see that admissibility implies $\left\|(s I-A)^{-1} B\right\| \leq \frac{M}{\sqrt{\operatorname{Re}(s)}}$. In Weiss [17] the following conjecture appeared.

Conjecture 1.3 Let $B \in \mathcal{L}\left(U, D\left(A^{*}\right)^{\prime}\right)$. Then the following statements are equivalent.

(i) $B$ is an admissible control operator for $T(\cdot)$.

(ii) There exist constants $K, \omega>0$ such that

$$
\left\|(s I-A)^{-1} B\right\| \leq \frac{K}{\sqrt{\operatorname{Re}(s)}}, \quad s \in \mathbb{C}, \quad \operatorname{Re}(s)>\omega .
$$

It is known [6] that this conjecture is also true for left-invertible semigroups, as well as for normal analytic ones. Since Weiss [17] showed that Conjecture 1.3 implies Conjecture 1.2, we have disproved Conjecture 1.3 as well. We also show that our example from Section 2 satisfies the stronger necessary condition for admissibility given in Staffans [14, Section 4.2], namely,

$$
\left\|(s I-A)^{-n} B\right\| \leq M \frac{1}{n^{1 / 4} \operatorname{Re}(s)^{n-1 / 2}}, \quad n \in \mathbb{N}, \quad s \in \mathbb{C}, \quad \operatorname{Re}(s)>\omega .(12)
$$

A necessary and sufficient condition for admissibility was obtained by Grabowski and Callier [5]. They showed that $B$ is an admissible control operator for $T(\cdot)$ 
if and only if there exist positive $M$ and $\omega$ such

$$
\left\|\sum_{k=0}^{n-1} \frac{e^{-s(\cdot)}(\cdot)^{k}}{k !} B^{*}\left(s I-A^{*}\right)^{-(n-k)} x_{0}\right\|_{L^{2}(0, \infty ; U)} \leq \frac{M}{\operatorname{Re}(s)^{n}}\left\|x_{0}\right\|
$$

for all $n \in \mathbb{N}, x_{0} \in H$, and all $s$ with $\operatorname{Re}(s)>\omega$. However, this condition is very hard to check. We remark that since this condition was obtained via the Hille-Yosida theorem, it is sufficient to check (13) for real $s$ only.

If we study the limit behavior of solutions of (7), a stronger concept than admissibility is needed, called infinite-time admissibility.

Definition 1.4 $B \in \mathcal{L}\left(U, D\left(A^{*}\right)^{\prime}\right)$ is called an infinite-time admissible control operator for $T(\cdot)$, if there exists a constant $M>0$ such that

$$
\left\|\int_{0}^{\infty} T(\rho) B u(\rho) d \rho\right\|_{H} \leq M\|u\|_{L^{2}(0, \infty ; U)}, \quad u \in L^{2}(0, \infty ; U) .
$$

$B \in \mathcal{L}\left(U, D\left(A^{*}\right)^{\prime}\right)$ is a weakly infinite-time admissible control operator for $T(\cdot)$ if, for every $v \in U, B v$ is an infinite-time admissible operator for $T(\cdot)$.

Of course, every infinite-time admissible control operator for $T(\cdot)$ is an admissible control operator for $T(\cdot)$, and if $T(\cdot)$ is exponentially stable, then admissibility and infinite-time admissibility are equivalent notions (as shown in [16]). In Section 3 we give an example showing that in general admissibility together with weak infinite-time admissibility does not imply infinite-time admissibility. In this example $A$ and $B$ are bounded and even compact so that $T(\cdot)$ is a uniformly continuous (semi)group. Moreover, in this example $T(\cdot)$ is analytic, bounded and strongly stable. The same example shows that in general the condition

$$
\left\|(s I-A)^{-1} B\right\| \leq \frac{K}{\sqrt{\operatorname{Re}(s)}}, \quad s \in \mathbb{C}, \quad \operatorname{Re}(s)>0,
$$

for some $K>0$, does not imply the infinite-time admissibility of $B$. In Jacob, Partington and Pott [8] it has been shown that weak infinite-time admissibility does not imply infinite-time admissibility even if the semigroup is a contraction semigroup.

In the last section we convert our counterexample into an example which demonstrates that for an infinitesimal generator $A$ the estimate

$$
\left\|(s I-A)^{-1}\right\| \leq \frac{M}{\operatorname{Re}(s)}, \quad s \in \mathbb{C}, \quad \operatorname{Re}(s)>0,
$$


does not imply the boundedness of the $C_{0}$-semigroup $T(\cdot)$. Note that this estimate is the first estimate in the Hille-Yosida theorem. Although it is known that this estimate is not sufficient to conclude that $A$ is the infinitesimal generator of a $C_{0}$-semigroup on the Hilbert space $H$, one could hope that if $A$ is the infinitesimal generator of a $C_{0}$-semigroup, then this would be sufficient to conclude the uniform boundedness of the semigroup.

Note that if $A$ is the infinitesimal generator of a $C_{0}$-semigroup which satisfies for some $\gamma \in[0,1)$

$$
\left\|(s I-A)^{-1}\right\| \leq \frac{M}{\operatorname{Re}(s)^{\gamma}}
$$

for all $s$ with $\operatorname{Re}(s)>0$, then the semigroup is exponentially stable (see Staffans [14, Lemma 3.11.7]).

\section{Weak admissibility does not imply admissibility}

Throughout this paper $H$ is a separable Hilbert space. All our examples are based on the fact that it is possible to find a normalized basis $\left\{\varphi_{n}\right\}_{n \in \mathbb{N}}$ in $H$ which is not Hilbertian. By a basis we mean a sequence $\left\{\varphi_{n}\right\}_{n \in \mathbb{N}}$ such that for every $x \in H$ there exists a unique sequence of scalar coefficients $\left\{f_{n}\right\}_{n \in \mathbb{N}}$, such that

$$
x=\sum_{n=1}^{\infty} f_{n} \varphi_{n}:=\lim _{N \rightarrow \infty} \sum_{n=1}^{N} f_{n} \varphi_{n} .
$$

This basis is normalized if $\left\|\varphi_{n}\right\|=1$ for all $n \in \mathbb{N}$. Such bases are studied in great detail in Singer [13]. A basis $\left\{\varphi_{n}\right\}_{n \in \mathbb{N}}$ is Hilbertian if there is a constant $C$ such that for all $N \in \mathbb{N}$ and all scalar sequences $\left\{f_{n}\right\}_{n=1}^{N}$ we have

$$
\left\|\sum_{n=1}^{N} f_{n} \varphi_{n}\right\|^{2} \leq C \sum_{n=1}^{N}\left|f_{n}\right|^{2} .
$$

The semigroups that we construct and their generators will be diagonal operators with respect to some basis. Such operators have been studied by several people. The following nice result is well-known, and a proof can be found in e.g. Benamara and Nikolski [1, Lemma 3.2.5].

Lemma 2.1 Let $\left\{\varphi_{n}\right\}_{n \in \mathbb{N}}$ be a basis of $H$, and let $\left\{q_{n}\right\}_{n \in \mathbb{N}}$ be a sequence of 
scalars. For each $N \in \mathbb{N}$ and scalar sequence $\left\{f_{n}\right\}_{n=1}^{N}$, define

$$
Q \sum_{n=1}^{N} f_{n} \varphi_{n}=\sum_{n=1}^{N} q_{n} f_{n} \varphi_{n} .
$$

If the total variation of the sequence $\left\{q_{n}\right\}_{n \in \mathbb{N}}$ is finite, i.e., if

$$
\operatorname{Var}(q):=\sum_{n=1}^{\infty}\left|q_{n+1}-q_{n}\right|<\infty
$$

then $Q$ can be extended to a bounded linear operator on $H$, and

$$
\|Q\| \leq K\left(\operatorname{Var}(q)+\limsup _{n \rightarrow \infty}\left|q_{n}\right|\right)
$$

where $K$ is a constant independent of the sequence $q$.

In order to calculate the total variation, the following observation is useful. If $f$ is a continuous function which is non-decreasing or non-increasing on the interval $(a, b)$, and if the sequence $\left\{q_{n}\right\}_{n \in \mathbb{N}} \subset(a, b)$ is non-decreasing or non-increasing, then $\operatorname{Var}(f(q)) \leq|f(a)-f(b)|$.

Another ingredient in our counterexamples are Carleson measures. Let $\mathbb{C}_{+}$ denote the open right half plane, i.e., $\mathbb{C}_{+}:=\{s \in \mathbb{C} \mid \operatorname{Re}(s)>0\}$. A positive measure $\sigma$ in $\mathbb{C}_{+}$is a Carleson measure if there exists a constant $m$ such that

$$
\sigma(Q) \leq m h
$$

for all squares $Q=\left\{s=s_{r}+i s_{i} \in \mathbb{C}_{+} \mid 0<s_{r}<h, y_{0}<s_{i}<y_{0}+h\right\}$.

These measures play an important role in the theory of complex interpolation problems, see Garnett [4]. We use one of these results for the Hardy spaces $H^{p}$. The Hardy space $H^{p}, p \geq 1$, is defined as the space of all functions which are holomorphic on $\mathbb{C}_{+}$and for which

$$
\sup _{r>0} \int_{-\infty}^{\infty}|f(r+i \omega)|^{p} d \omega<\infty .
$$

$H^{p}$ is a Banach space with its norm given by the $p$ th root of the expression in (15). The following result relates $H^{p}$ with Carleson measures. For the proof we refer to Garnett [4, Theorem II.3.9]. 
Lemma 2.2 If $\sigma$ is a Carleson measure, then

$$
\int|f|^{p} d \sigma \leq A\|f\|_{H^{p}}^{p}, \quad f \in H^{p}
$$

More information on Carleson measures can be found in Garnett [4].

We are now in a position to present the example showing that weak admissibility of $B$ for $T(\cdot)$ does not imply admissibility.

Example 2.3 As in all our examples, we let $H$ be a separable Hilbert space, and let $\left\{\varphi_{n}\right\}_{n \in \mathbb{N}}$ be a normalized basis for $H$ which is not Hilbertian. Examples of such bases can be found in Singer [13, page 428]. We let $\left\{\mu_{n}\right\}_{n \in \mathbb{N}} \subset$ $(1, \infty)$ be a monotonically increasing sequence with $\lim _{n \rightarrow \infty} \mu_{n}=\infty$ such that $\sum_{n=1}^{\infty} \mu_{n} \delta_{\mu_{n}}$ is a Carleson measure. We may choose $\mu_{n}:=2^{n}$, see Garnett [4, page 288], but other choices are also possible.

First we construct a $C_{0}$-semigroup on $H$. For all $t \geq 0$ and $x \in H$ of the form $x=\sum_{n=1}^{N} f_{n} \varphi_{n}$ we define

$$
T(t) \sum_{n=1}^{N} f_{n} \varphi_{n}:=\sum_{n=1}^{N} e^{-\mu_{n} t} f_{n} \varphi_{n}
$$

Since the sequence $\left\{\mu_{n}\right\}_{n}$ is monotonically increasing and since $\lim _{n \rightarrow \infty} \mu_{n}=$ $\infty$, we get by Lemma 2.1 that $T(t)$ has a linear bounded extension to $H$. Thus $T(t) \in \mathcal{L}(H)$, and

$$
\|T(t)\| \leq K e^{-t}, \quad t \geq 0
$$

Clearly, $T(0)=I$ and $T(t) T(s)=T(t+s)$ for $t, s \geq 0$. We will show that $T(\cdot)$ is strongly continuous. For each $x \in H$, there exists a sequence $\left\{f_{n}\right\}_{n \in \mathbb{N}}$ of scalars such that (14) holds. Choose $\varepsilon>0$ and choose $N$ such that $\left\|x-x_{N}\right\|_{H}<\varepsilon$, where $x_{N}:=\sum_{n=1}^{N} f_{n} \varphi_{n}$. Next choose $t_{0}>0$ such that $\sum_{n=1}^{N}\left|e^{-\mu_{n} t_{0}}-1\right|\left|f_{n}\right| \leq$ $\varepsilon$. Then we have for $t \in\left(0, t_{0}\right)$ that

$$
\begin{aligned}
\|T(t) x-x\| & \leq\left\|T(t) x-T(t) x_{N}\right\|+\left\|T(t) x_{N}-x_{N}\right\|+\left\|x_{N}-x\right\| \\
& \leq K \varepsilon+\sum_{n=1}^{N}\left|e^{-\mu_{n} t_{0}}-1\right|\left|f_{n}\right|+\varepsilon \leq[K+2] \varepsilon .
\end{aligned}
$$

Thus $T(\cdot)$ is a $C_{0}$-semigroup on $H$. This $C_{0}$-semigroup was also used by Le Merdy [10] to show that there exists a uniformly bounded, compact $C_{0^{-}}$ semigroup which is not equivalent to a contraction semigroup. Let $A$ denote 
the infinitesimal generator of $T(\cdot)$. It is easy to see that

$$
A \varphi_{n}=-\mu_{n} \varphi_{n}, \quad n \in \mathbb{N} .
$$

We show that $T(\cdot)$ is analytic. Since $T(\cdot)$ is uniformly bounded, it is sufficient (see [11, Theorem 2.5.2]) to show that

$$
\left\|(s I-A)^{-1}\right\| \leq \frac{M}{|\operatorname{Im} s|}, \quad s \in \mathbb{C}_{+} .
$$

Let $s=s_{r}+i s_{i} \in \mathbb{C}_{+}$. Clearly, $(s I-A)^{-1} \varphi_{n}=\frac{1}{s+\mu_{n}} \varphi_{n}$, for $n \in \mathbb{N}$. In order to prove (18), we first estimate the variation of the sequence $\gamma_{n}:=\frac{1}{s+\mu_{n}}$. For each $n \in \mathbb{N}$ we have

$$
\begin{aligned}
\left|\gamma_{n+1}-\gamma_{n}\right| & =\left|\frac{1}{s+\mu_{n+1}}-\frac{1}{s+\mu_{n}}\right|=\left|\int_{-\mu_{n+1}}^{-\mu_{n}} \frac{d}{d x} \frac{1}{s-x} d x\right| \\
& =\left|\int_{-\mu_{n+1}}^{-\mu_{n}} \frac{1}{(s-x)^{2}} d x\right| \leq \int_{-\mu_{n+1}}^{-\mu_{n}} \frac{1}{|s-x|^{2}} d x
\end{aligned}
$$

Thus

$$
\begin{aligned}
\operatorname{Var}(\gamma) & \leq \int_{-\infty}^{-1} \frac{1}{|s-x|^{2}} d x=\int_{-\infty}^{-1} \frac{1}{\left|s_{i}\right|^{2}+\left|x-s_{r}\right|^{2}} d x \\
& \leq \int_{-\infty}^{0} \frac{1}{\left|s_{i}\right|^{2}+|x|^{2}} d x=\frac{\pi}{2\left|s_{i}\right|} .
\end{aligned}
$$

Using Lemma 2.1 we get the following estimate for $\left\|(s I-A)^{-1}\right\|$ :

$$
\left\|(s I-A)^{-1}\right\| \leq K\left(\operatorname{Var}(\gamma)+\left|\lim _{n \rightarrow \infty} \gamma_{n}\right|\right) \leq \frac{K \pi}{2|\operatorname{Im} s|},
$$

where $K>0$ is independent of $s$. Thus $T(\cdot)$ is analytic.

Next we construct an operator $B$ which is weakly admissible but not admissible. We choose $U=\ell^{2}(\mathbb{N})$ and for each finite sequence $\left\{\nu_{n}\right\}_{n=1}^{N}$, we define

$$
B\left\{\nu_{n}\right\}_{n=1}^{N}:=\sum_{n=1}^{N} \sqrt{\mu_{n}} \nu_{n} \varphi_{n} .
$$


Since $D\left(A^{*}\right)^{\prime}$ is the completion of $H$ with respect to the norm

$$
\|x\|_{D\left(A^{*}\right)^{\prime}}=\left\|A^{-1} x\right\|_{H},
$$

it is easy to see that $B$ can be extended to an operator in $\mathcal{L}\left(U, D\left(A^{*}\right)^{\prime}\right)$.

Next we prove that $B$ is only weakly admissible.

(i) $B$ is not an admissible control operator for $T(\cdot)$.

Since $T(\cdot)$ is exponentially stable, it is enough to show that $B$ is not an infinite-time admissible control operator for $T(\cdot)$.

Choose an arbitrary finite scalar sequence $\left\{\nu_{n}\right\}_{n=1}^{N}$ and define

$$
u_{N}(t):=\sum_{n=1}^{N} \sqrt{\mu_{n}} \nu_{n} e_{n} e^{-\mu_{n} t}, \quad t \geq 0
$$

where $\left\{e_{n}\right\}_{n \in \mathbb{N}}$ is the standard basis of $\ell^{2}(\mathbb{N})$, i.e., $\left(e_{n}\right)_{k}=1$ if $k=n$ and $\left(e_{n}\right)_{k}=0$ otherwise. Clearly,

$$
\left\|u_{N}\right\|_{L^{2}\left(0, \infty ; \ell^{2}(\mathbb{N})\right)}^{2}=\sum_{n=1}^{N} \mu_{n}\left|\nu_{n}\right|^{2} \int_{0}^{\infty} e^{-2 \mu_{n} t} d t=\frac{1}{2} \sum_{n=1}^{N}\left|\nu_{n}\right|^{2} .
$$

Furthermore, using the fact that $B e_{n}=\sqrt{\mu_{n}} \varphi_{n}$ and $T(t) \varphi_{n}=e^{-\mu_{n} t} \varphi_{n}$, we see that

$$
\int_{0}^{\infty} T(\tau) B u_{N}(\tau) d \tau=\int_{0}^{\infty} \sum_{n=1}^{N} \mu_{n} \nu_{n} e^{-2 \mu_{n} \tau} \varphi_{n} d \tau=\frac{1}{2} \sum_{n=1}^{N} \nu_{n} \varphi_{n} .
$$

If $B$ was infinite-time admissible, then there would exist a constant $M>0$ such that

$$
\left\|\int_{0}^{\infty} T(\tau) B u(\tau) d \tau\right\|_{H} \leq M\|u\|_{L^{2}\left(0, \infty ; \ell^{2}(\mathbb{N})\right)}, \quad u \in L^{2}\left(0, \infty ; \ell^{2}(\mathbb{N})\right) .
$$

Combining this estimate with the equalities (19) and (20) we get

$$
\begin{aligned}
\left\|\sum_{n=1}^{N} \nu_{n} \varphi_{n}\right\|^{2} & =4\left\|\int_{0}^{\infty} T(\tau) B u_{N}(\tau) d \tau\right\|^{2} \\
& \leq 4 M^{2}\left\|u_{N}\right\|_{L^{2}\left(0, \infty ; \ell^{2}(\mathbb{N})\right)}^{2}=2 M^{2} \sum_{n=1}^{N}\left|\nu_{n}\right|^{2} .
\end{aligned}
$$

However, this inequality says that the basis $\left\{\varphi_{n}\right\}_{n \in \mathbb{N}}$ is Hilbertian, contrary to our assumption. Thus, $B$ is not an admissible control operator for $T(\cdot)$. 
(ii) $B$ is a weakly admissible control operator for $T(\cdot)$ i.e., for every $v \in \ell^{2}(\mathbb{N})$, $B v$ is an infinite-time admissible control operator for $T(\cdot)$.

Let $v=\left\{\nu_{n}\right\}_{n \in \mathbb{N}} \in \ell^{2}(\mathbb{N})$, and let $v_{N}:=\left\{\nu_{n}\right\}_{n=1}^{N}$. For $u \in L^{2}(0, \infty)$ we get

$$
\begin{aligned}
\left\|\int_{0}^{\infty} T(\tau) B v_{N} u(\tau) d \tau\right\|_{H} & =\left\|\int_{0}^{\infty} \sum_{n=1}^{N} e^{-\mu_{n} \tau} \nu_{n} \sqrt{\mu_{n}} \varphi_{n} u(\tau) d \tau\right\|_{H} \\
& =\left\|\sum_{n=1}^{N} \nu_{n} \sqrt{\mu_{n}} \int_{0}^{\infty} e^{-\mu_{n} \tau} u(\tau) d \tau \varphi_{n}\right\|_{H} \\
& =\left\|\sum_{n=1}^{N} \nu_{n} \sqrt{\mu_{n}} \hat{u}\left(\mu_{n}\right) \varphi_{n}\right\|_{H} \\
& \leq \sum_{n=1}^{N}\left|\nu_{n} \sqrt{\mu_{n}} \hat{u}\left(\mu_{n}\right)\right| \\
& \leq\left(\sum_{n=1}^{N}\left|\nu_{n}\right|^{2}\right)^{1 / 2}\left(\sum_{n=1}^{N}\left|\sqrt{\mu_{n}} \hat{u}\left(\mu_{n}\right)\right|^{2}\right)^{1 / 2} \\
& \leq M_{2}\left\|v_{N}\right\|\|\hat{u}\|_{H^{2}},
\end{aligned}
$$

where we have used Lemma 2.2 and the fact that the Laplace transform of any $L^{2}(0, \infty)$ function lies in $H^{2}$. Since $\|\hat{u}\|_{H_{2}}^{2}=2 \pi\|u\|_{L^{2}(0, \infty)}^{2}$, we have shown that $B v_{N}$ is an admissible control operator for $T(\cdot)$.

For $N \rightarrow \infty$, we have that $B v_{N} \rightarrow B v$ in $D\left(A^{*}\right)^{\prime}$, and hence

$$
\int_{0}^{\infty} T(\tau) B v_{N} u(\tau) d \tau \rightarrow \int_{0}^{\infty} T(\tau) B v u(\tau) d \tau \text { in } D\left(A^{*}\right)^{\prime}
$$

On the other hand, (22) shows that the sequence $\int_{0}^{\infty} T(\tau) B v_{N} u(\tau) d \tau$ is bounded in $H$, which implies the convergences (23) also holds in the weak topology of $H$. By the weak compactness of the unit ball in $H$, $\int_{0}^{\infty} T(\tau) \operatorname{Bvu}(\tau) d \tau \in H$ and

$$
\left\|\int_{0}^{\infty} T(\tau) B v u(\tau) d \tau\right\|_{H} \leq M_{3}\|v\|\|u\|_{L^{2}(0, \infty)} .
$$

Thus, $B v$ is an admissible control operator for $T(\cdot)$.

If $B$ is weakly admissible, then it is not hard to show that $B v$ satisfies the the sequence of estimates (12), with $M=M_{v}$, see [14, Section 4.2]. Using the uniform boundedness theorem, we conclude that every weakly admissible $B$ satisfies (12). Hence the previous example shows that this condition is not sufficient for admissibility. Yet we will show that the input operator from Example 2.3 satisfies a stronger sequence of estimates, and thus showing that 
this stronger sequence of estimates is not sufficient either. Here we explicitly use the fact that $\mu_{n}=2^{n}$.

Let $s$ be an element of $\mathbb{C}_{+}$, and let $v=\left\{v_{k}\right\}_{k=1}^{\infty} \in \ell^{2}(\mathbb{N})$ have norm one. We have the following estimate

$$
\begin{aligned}
\left\|(s I-A)^{-n} B v\right\|^{2} & =\left|\sum_{k=1}^{\infty} \frac{\sqrt{2^{k}}}{\left(s+2^{k}\right)^{n}} v_{k} \varphi_{k}\right|^{2} \leq\left(\sum_{k=1}^{\infty} \frac{\sqrt{2^{k}}}{\left(\operatorname{Re}(s)+2^{k}\right)^{n}}\left|v_{k}\right|\right)^{2} \\
& \leq \sum_{k=1}^{\infty} \frac{2^{k}}{\left(\operatorname{Re}(s)+2^{k}\right)^{2 n}}
\end{aligned}
$$

where we have used the Cauchy-Schwarz inequality. In order to estimate this last expression, we introduce the monotonically decreasing sequence $a_{k}:=$ $\frac{1}{(\operatorname{Re}(s)+k)^{2 n}}$. Then for $N \geq 2^{K}$ we have

$$
\begin{aligned}
\sum_{k=1}^{N} a_{k} & \geq a_{1}+a_{2}+\left(a_{3}+a_{4}\right)+\cdots+\left(a_{2^{K-1}+1}+\cdots+a_{2^{K}}\right) \\
& \geq a_{2}+2 a_{4}+\cdots+2^{K-1} a_{2^{K}}=\frac{1}{2} \sum_{k=1}^{K} 2^{k} a_{2^{k}},
\end{aligned}
$$

and so

$$
\sum_{k=1}^{\infty} \frac{2^{k}}{\left(\operatorname{Re}(s)+2^{k}\right)^{2 n}} \leq 2 \sum_{k=1}^{\infty} \frac{1}{(\operatorname{Re}(s)+k)^{2 n}}
$$

Using this in our estimate of $\left\|(s I-A)^{-n} B v\right\|$, we obtain that

$$
\begin{aligned}
\left\|(s I-A)^{-n} B v\right\|^{2} & \leq 2 \sum_{k=1}^{\infty} \frac{1}{(\operatorname{Re}(s)+k)^{2 n}} \\
& \leq 2 \int_{0}^{\infty} \frac{1}{(\operatorname{Re}(s)+t)^{2 n}} d t \leq \frac{2}{2 n-1} \frac{1}{(\operatorname{Re}(s))^{2 n-1}} .
\end{aligned}
$$

\section{Admissibility and weak infinite-time admissibility does not imply infinite-time admissibility}

In the previous section we have shown that weak admissibility does not imply admissibility. From (24) we see that the input operator in our example is weakly infinite-time admissible. Hence we already proved that weak infinitetime admissibility does not imply admissibility, and thus not infinite-time 
admissibility either. However, if $B$ is admissibble, would weak infinite-time admissibility imply infinite-time admissibility? In the next example we show that this does not hold either. Note, that in this example the operators $A$ and $B$ are compact elements of $\mathcal{L}(H)$ and $\mathcal{L}\left(\ell^{2}(\mathbb{N}), H\right)$, respectively and that $T(\cdot)$ is bounded and strongly stable. In particular, $B$ is an admissible control operator for $A$. Furthermore, note that the operator $A$ in this example is the inverse of the operator $A$ in the previous example.

Example 3.1 Let $\left\{\lambda_{n}\right\}_{n \in \mathbb{N}} \subset(0,1)$ be a monotonically decreasing sequence with $\lim _{n \rightarrow \infty} \lambda_{n}=0$, and such that $\sum_{n=1}^{\infty} \lambda_{n} \delta_{\lambda_{n}}$ is a Carleson measure on the right half plane $\mathbb{C}_{+}$. We could for example choose $\lambda_{n}:=2^{-n}$, see Garnett $[4$, page 288]. We take $\left\{\varphi_{n}\right\}_{n \in \mathbb{N}}$ to be the same as in Example 2.3.

We now define $A$ by

$$
A \varphi_{n}=-\lambda_{n} \varphi_{n}, \quad n \in \mathbb{N}
$$

Since the sequence $\left\{\lambda_{n}\right\}_{n}$ is monotonically decreasing it is easy to see that $\left\{\lambda_{n}\right\}_{n \in \mathbb{N}}$ is of bounded variation. Now by Lemma 2.1, we get that $A$ has a bounded linear extension to $H$, that is $A \in \mathcal{L}(H)$. Let $T(\cdot)$ be the $C_{0^{-}}$ semigroup generated by $A$, that is

$$
T(t) \varphi_{n}=e^{-\lambda_{n} t} \varphi_{n}, \quad t \geq 0, \quad n \in \mathbb{N} .
$$

The operators $A$ and $T(\cdot)$ have some nice properties.

(i) $T(\cdot)$ is bounded and strongly stable.

Proof: By Lemma 2.1 we have for $t \geq 0$

$$
\|T(t)\| \leq 2 K
$$

and thus the $C_{0}$-semigroup $T(\cdot)$ is bounded.

Next we show that $T(\cdot)$ is strongly stable. Let $x=\sum_{n=1}^{\infty} f_{n} \varphi_{n} \in H$ and $\varepsilon>0$. Then there exists an $N \in \mathbb{N}$ such that $x_{N}:=\sum_{n=1}^{N} f_{n} \varphi_{n}$ satisfies

$$
\left\|x-x_{N}\right\|_{H}<\varepsilon .
$$

Thus for sufficiently large $t>0$ we have

$$
\begin{aligned}
\|T(t) x\| & \leq\left\|T(t) x-T(t) x_{N}\right\|+\left\|T(t) x_{N}\right\| \\
& \leq\|T(t)\|\left\|x-x_{N}\right\|+\left\|\sum_{n=1}^{N} e^{-\lambda_{n} t} f_{n} \varphi_{n}\right\| \\
& \leq 2 K \varepsilon+\sum_{n=1}^{N} e^{-\lambda_{n} t}\left|f_{n}\right|\left\|\varphi_{n}\right\| \leq 2 K \varepsilon+\varepsilon,
\end{aligned}
$$


and so $T(\cdot)$ is strongly stable.

(ii) The operator $A$ is compact.

Proof: Define $A_{N}, N \in \mathbb{N}$, by

$$
A_{N} \varphi_{n}= \begin{cases}-\lambda_{k} \varphi_{n}, & n \leq N \\ 0, & n>N\end{cases}
$$

Clearly, $A_{N}$ has rank $N$. Using Lemma 2.1, we get the estimate

$$
\left\|A-A_{N}\right\| \leq 2 K \lambda_{N+1} \rightarrow 0 \text { as } N \rightarrow \infty,
$$

which shows that $A$ is compact.

Next we define the control operator $B$. We again choose $U=\ell^{2}(\mathbb{N})$, and for every finite sequence $\left\{\nu_{n}\right\}_{n=1}^{N}$ we define

$$
B\left\{\nu_{n}\right\}_{n=1}^{N}=\sum_{n=1}^{N} \sqrt{\lambda_{n}} \nu_{n} \varphi_{n}
$$

(iii) $B$ can be extended to a bounded linear operator.

Proof: We have

$$
\begin{aligned}
\left\|B\left\{\nu_{n}\right\}_{n=1}^{N}\right\| & =\left\|\sum_{n=1}^{N} \sqrt{\lambda_{n}} \nu_{n} \varphi_{n}\right\| \leq \sum_{n=1}^{N} \sqrt{\lambda_{n}}\left|\nu_{n}\right|\left\|\varphi_{n}\right\| \\
& =\sum_{n=1}^{N} \sqrt{\lambda_{n}}\left|\nu_{n}\right| \leq \sqrt{\left(\sum_{n=1}^{N} \lambda_{n}\right) \sum_{n=1}^{N}\left|\nu_{n}\right|^{2}}
\end{aligned}
$$

Thus we have that

$$
\left\|B\left\{\nu_{n}\right\}_{n=1}^{N}\right\|^{2} \leq\left(\sum_{n=1}^{N} \lambda_{n}\right) \sum_{n=1}^{N}\left|\nu_{n}\right|^{2} \leq C\left\|\left\{\nu_{n}\right\}_{n=1}^{N}\right\|_{\ell^{2}(\mathbb{N})}^{2},
$$

where $C:=\sum_{n=1}^{\infty} \lambda_{n}<\infty$, since $\sum_{n=1}^{\infty} \lambda_{n} \delta_{\lambda_{n}}$ is a Carleson measure with support in $(0,1)$. This shows that $B$ has a bounded linear extension, i.e., $B \in \mathcal{L}\left(\ell^{2}(\mathbb{N}), H\right)$. In particular, $B$ is an admissible control operator for $T(\cdot)$.

(iv) The operator $B$ is compact. This is shown similar as in Part ii.

(v) $B$ is not an infinite-time admissible control operator for $T(\cdot)$. This is shown in the same way as in Example 2.3.

(vi) Similarly, as in Example 2.3, we can show that for every $v \in U, B v$ is an infinite-time admissible control operator for $T(\cdot)$. Furthermore, as in (22) and (24) we have that

$$
\left\|\int_{0}^{\infty} T(\tau) B v u(\tau) d \tau\right\| \leq M_{2}\|v\|\|u\|_{L^{2}(0, \infty)} .
$$


(vii) There exists a constant $K>0$ such that

$$
\left\|(s I-A)^{-1} B\right\| \leq \frac{K}{\sqrt{\operatorname{Re}(s)}}, \quad s \in \mathbb{C}, \operatorname{Re}(s)>0 .
$$

Proof: Substituting $u(\tau)=e^{-s \tau}$ in (26), where $\operatorname{Re}(s)>0$ gives that

$$
\left\|(s I-A)^{-1} B v\right\| \leq \frac{M_{2}\|v\|}{\sqrt{\operatorname{Re}(s)}}
$$

Since this holds for all $v \in H$ we have proved the assertion.

\section{A semigroup example}

A direct consequence of the Hille-Yosida theorem is that a $C_{0}$-semigroup $T_{e}(t)$ is uniformly bounded if and only if there exists a constant $M$ such that its generator $A_{e}$ satisfies

$$
\left\|\left(s I-A_{e}\right)^{-n}\right\| \leq \frac{M}{\operatorname{Re}(s)^{n}} \quad \text { for all } n \in \mathbb{N} \text { and } s \in \mathbb{C}_{+} .
$$

If $T_{e}(t)$ is a $C_{0}$-semigroup on the Hilbert space $H_{e}$, then its growth bound is negative if and only if $\left(s I-A_{e}\right)^{-1}$ is uniformly bounded in the open right halfplane. Motivated by this result, one wonders if the first inequality of the HilleYosida theorem is sufficient to determine the exact growth of the semigroup. More precisely, suppose that the infinitesimal generator $A_{e}$ satisfies

$$
\left\|\left(s I-A_{e}\right)^{-1}\right\| \leq \frac{M}{\operatorname{Re}(s)} \quad \text { for all } s \in \mathbb{C}_{+},
$$

does this imply that $T_{e}(t)$ is uniformly bounded?

Using the example of the previous section we show that this is in general not true, by constructing a $C_{0}$-semigroup for which the infinitesimal generator $A_{e}$ satisfies (27) but

$$
\lim _{t \rightarrow \infty}\left\|T_{e}(t)\right\|=\infty
$$

We remark that the estimate (27) implies that $\|T(t)\| \leq M(1+t)$, but we do not know how sharp this estimate is. 
Furthermore, we would like to remark that if there exists a $\gamma \in[0,1)$ such that the infinitesimal generator $A_{e}$ satisfies

$$
\left\|\left(s I-A_{e}\right)^{-1}\right\| \leq \frac{M}{\operatorname{Re}(s)^{\gamma}} \quad \text { for all } s \in \mathbb{C}_{+},
$$

then the $C_{0}$-semigroup is exponentially stable, see [14, Lemma 3.11.7].

Consider the operators $A$ and $B$ of Example 3.1, and let $T(\cdot)$ denote the bounded semigroup generated by $A$. With these operators we define the semigroup $T_{e}(t)$ on $H \oplus L^{2}\left(0, \infty ; \ell^{2}(\mathbb{N})\right)$ as

$$
T_{e}(t)\left(\begin{array}{c}
x \\
f
\end{array}\right)=\left(\begin{array}{c}
T(t) x+\int_{0}^{t} T(t-\tau) B f(\tau) d \tau \\
f(t+\cdot)
\end{array}\right), \quad t \geq 0 .
$$

$B$ is a bounded operator, and hence $B$ is an admissible control operator for $T(\cdot)$. According to Engel [3], this implies that $T_{e}(t)$ is a $C_{0}$-semigroup on $H \oplus L^{2}\left(0, \infty ; \ell^{2}(\mathbb{N})\right)$. Since $B$ is not infinite-time admissible we know that $T_{e}(t)$ cannot be a bounded semigroup. However, we will show that its infinitesimal generator satisfies (27).

By taking the Laplace transform of the semigroup, we see that the resolvent is given by

$$
\left(s I-A_{e}\right)^{-1}\left(\begin{array}{l}
x \\
f
\end{array}\right)=\left(\begin{array}{c}
(s I-A)^{-1} x+(s I-A)^{-1} B \hat{f}(s) \\
\widehat{f(t+\cdot)}(s)
\end{array}\right)
$$

Since the left shift is a contraction semigroup we know that $L^{2}\left(0, \infty ; \ell^{2}(\mathbb{N})\right)$ norm of $\widehat{f(t+\cdot)}(s)$ is bounded by $\|f\|$ times $1 / \operatorname{Re}(s)$. Furthermore, since $T(\cdot)$ is a bounded semigroup, a similar estimate holds for $(s I-A)^{-1}$. Thus we have that for $s \in \mathbb{C}_{+}$

$$
\begin{aligned}
\left\|\left(s I-A_{e}\right)^{-1}\left(\begin{array}{l}
x \\
f
\end{array}\right)\right\|^{2} \leq & \frac{2 M^{2}}{\operatorname{Re}(s)^{2}}\|x\|^{2}+ \\
& 2\left\|(s I-A)^{-1} B\right\|^{2}\|\hat{f}(s)\|^{2}+\frac{1}{\operatorname{Re}(s)^{2}}\|f\|^{2} .
\end{aligned}
$$

Since $f \in L^{2}\left(0, \infty ; \ell^{2}(\mathbb{N})\right)$ we have that

$$
\|\hat{f}(s)\| \leq\|f\| / \sqrt{2 \operatorname{Re}(s)} \quad \text { for all } s \in \mathbb{C}_{+} .
$$


In Example 3.1 we proved the existence of a constant $K>0$ such that

$$
\left\|(s I-A)^{-1} B\right\| \leq K / \sqrt{\operatorname{Re}(s)} \quad \text { for all } s \in \mathbb{C}_{+} .
$$

Combining (29)-(31) gives that $A_{e}$ satisfies the estimate (27). Since the corresponding semigroup is unbounded, we have shown that the estimate (27) is not sufficient to conclude the boundedness of a $C_{0}$-semigroup.

\section{Acknowledgment}

The first author would like to thank Emmanuel Fricain for providing him with a copy of Singer [13] although he was extremely busy with the IWOTA-2000 conference.

\section{References}

[1] N.-E. Benamara and N. Nikolski. Resolvent test for similarity to a normal operator. Proc. Lond. Math. Soc., 78:585-626, 1999.

[2] R. F. Curtain and H. Zwart. An Introduction to Infinite-Dimensional Linear Systems Theory. Number 21 in Texts in Applied Mathematics. Springer Verlag, New York, 1995.

[3] K.-J. Engel. On the characterization of admissible control- and observation operators. Systems \& Control Letters 34:225-227, 1998.

[4] J. B. Garnett. Bounded Analytic Functions. Academic Press, 1981.

[5] P. Grabowski and F. M. Callier. Admissible observation operators, semigroup criteria of admissibility. Integral Equation and Operator Theory, 25:182-198, 1996.

[6] S. Hansen and G. Weiss. The operator Carleson measure criterion for admissibility of control operators for diagonal semigroups on $\ell_{2}$. Systems 83 Control Letters, 16:219-227, 1991.

[7] L. F. Ho and D. L. Russell. Admissible input elements for systems in Hilbert space and a Carleson measure criterion. SIAM J. Control Optim., 21(4):614640, 1983.

[8] B. Jacob, J.R. Partington, and S. Pott. Admissible and weakly admissible observation operators for the right shift semigroup. Submitted, 2000. 
[9] I. Lasiecka and R. Triggiani. Control Theory for Partial Differential Equations: Continuous and Approximation Theories, Volume I-II. Cambridge University Press, 2000.

[10] C. Le Merdy. A bounded compact semigroup on Hilbert space not similar to a contraction one. Progress in Nonlinear Differential Equations and Their Applications, 42:213-216, 2000.

[11] A. Pazy. Semigroups of Linear Operators and Applications to Partial Differential Equations Springer-Verlag, New York, 1983.

[12] D. Salamon. Infinite dimensional linear systems with unbounded control and observation: a functional analysis approach. Transactions of the American Mathematical Society, 300(2):383-431, 1987.

[13] I. Singer. Bases in Banach Spaces I. Springer-Verlag, Berlin, 1970.

[14] O.J. Staffans. Well-Posed Linear Systems, book manuscript (available at http://www.abo.fi/〜staffans).

[15] G. Weiss. Admissibility of input elements for diagonal semigroups on $l^{2}$. Systems \& Control Letters, 10:79-82, 1988.

[16] G. Weiss. Admissibility of unbounded control operators. SIAM J. Control Optim., 27:527-545, 1989.

[17] G. Weiss. Two conjectures on the admissibility of control operators. In W. Desch, F. Kappel, K. Kunisch, editors, Estimation and Control of Distributed Parameter Systems, pages 367-378, Birkhäuser Verlag, Basel, 1991. 